Philosophie ANTIQUE
Philosophie antique

Problèmes, Renaissances, Usages

$14 \mid 2014$

Le devoir

\title{
Maddalena BONELLI (éd.), Physique et métaphysique chez Aristote
}

Pinelopi Skarsouli

\section{OpenEdition}

\section{Journals}

Édition électronique

URL : https://journals.openedition.org/philosant/853

DOI : 10.4000/philosant.853

ISSN : 2648-2789

\section{Éditeur}

Éditions Vrin

\section{Édition imprimée}

Date de publication : 1 novembre 2014

Pagination : $346-349$

ISBN : 978-2-7574-0855-1

ISSN : $1634-4561$

Référence électronique

Pinelopi Skarsouli, « Maddalena Bonelıı (éd.), Physique et métaphysique chez Aristote », Philosophie antique [En ligne], 14 | 2014, mis en ligne le 01 novembre 2018, consulté le 04 décembre 2022. URL http://journals.openedition.org/philosant/853 ; DOI : https://doi.org/10.4000/philosant.853

\section{(c) (1) (9)}

Creative Commons - Attribution - Pas d'Utilisation Commerciale - Pas de Modification 4.0 International - CC BY-NC-ND 4.0

https://creativecommons.org/licenses/by-nc-nd/4.0/ 
the Intellect and with the Soul, but ascribe a proper demiurgic role only to the latter.

The book is generally well edited, although it contains a few typos and other mistakes: see, e.g., the use of italics on p. 6: "the speech Timaeus gives in the Timaeus"; 'Enn. IV 6 [8]' instead of 'Enn. IV 8 [6]' on p. 95, n. 63; and 'conditio' for 'creatio' on p. 136.

All in all, the volume succeeds in providing a rather unified series of papers, each of which contributes to illustrate the different approaches that have been taken in the Platonic tradition to understand the figure of the world-maker.

Alberto KOBEC

Katholieke Universiteit Leuven

Maddalena Bonelli (éd.), Physique et métaphysique chez Aristote, Paris, Librairie philosophique J. Vrin, 2012 (Bibliothèque d'histoire de la philosophie), 308 pages, ISBN 978-2-7116-2455-3

Les études recueillies dans cet ouvrage collectif sont issues, dans leur grande majorité, de communications et de débats dans le cadre du séminaire du « Centre Léon Robin de Recherches sur la Pensée Antique » (UMR 8061, Université de Paris IV-Sorbonne, CNRS et ENS), consacré, pendant les années 2005-2007, à la Physique et la Métaphysique chez Aristote.

Les contributions, rédigées ou traduites en français, sont soigneusement structurées et caractérisées par une argumentation claire et approfondie. Elles portent presque toutes sur ces deux questions importantes : 1) quel est le contenu de la science ou discipline qui sera appelée plus tard «métaphysique » ? On pourrait ajouter : est-il possible d'établir un lien entre les sujets métaphysiques à première vue disparates abordés dans le livre d'Aristote qui porte ce nom ? 2) Quel est plus exactement le rapport entre physique et métaphysique chez Aristote ?

Ainsi, après la préface de Maddalena Bonelli, les études sont présentées selon un ordre qui prend son point de départ dans l'Organon, en passant par la Métaphysique (qui constitue l'objet de la plus grande partie d'entre elles) pour arriver à la Physique, au De anima et aux ouvrages biologiques, tandis que la dernière contribution porte sur Alexandre d'Aphrodise. L'ouvrage se clôt par une bibliographie et trois index très utiles : sources, noms et notions.

Au tout début, la contribution de Curzio Chiesa (p. 19-37), intitulée « Le problème de l'être dans le De interpretatione (chapitre 11) », qui offre une analyse détaillée du traitement aristotélicien de la question du passage de « Homère est un poète » à « Homère est »; plus précisément, Aristote bloque ce genre d'inférence. Cela pose le problème de la différence entre « est » prédiqué « par accident » et « est » prédiqué « par soi ».

Stephen Menn, dans l'article suivant, intitulé « La sagesse comme science des quatre causes ?» (p. 39-68), se concentre sur la définition de la $\sigma \circ \phi i \alpha$ au livre A de la Métaphysique dont la leçon serait plutôt négative, à savoir que les prédécesseurs d'Aristote ont jusqu'à maintenant échoué à découvrir la sagesse, surtout parce qu'ils ont échoué à découvrir la bonne ảpxỷ et à y reconduire les choses comme à leur cause finale. Quant à la sagesse aristotélicienne, le livre A ne nous donne aucune raison de croire qu'elle sera une science de l'ensemble des quatre causes. En 
effet, l'étude en question démontre, en analysant plusieurs passages de la Métaphysique et de la Physique, que la sagesse sera pour Aristote la science des seules causes efficiente et finale, et plus précisément, la science des premières causes efficientes et finales, et des plus élevées. Cela lui permet de résoudre la première aporie du livre $\mathrm{B}$ et de répondre aux attentes concernant la sagesse que le livre A avait suscitées.

Les deux articles suivants portent sur la métaphysique en tant que science des êtres, en prolongeant ainsi la tentative de préciser l'objet qui est « au-delà » de la physique, ainsi que le statut et le contenu de la science qui s'en occupe. Jonathan Barnes, dans « La science des êtres » (p. 69-91), s'arrête sur la première phrase du livre $\Gamma$ de la Métaphysique et sur le sens de l'expression « être en tant qu'être ». La question qui se pose est la suivante : en quoi consisterait l'étude de l'être en tant qu'être ? Quel pourrait être le contenu d'une telle science ? Une première réponse serait que la science des êtres s'intéresse à ce qui appartient aux êtres en eux-mêmes et s'efforce de présenter sous une forme systématique les vérités qui en découlent. Cependant, si l'on considère les trois critères d'une preuve établis dans les Seconds Analytiques, la science des êtres devient très problématique, car il est extrêmement difficile d'en déterminer le genre, ainsi que les caractéristiques ou accidents essentiels. Le reste de l'article démontre de quelle façon on pourrait, malgré tout, défendre la conception aristotélicienne de la science en question. L'article de Juliette Lemaire, «Aristote : contradiction et métaphysique » (p.93-112), se concentre quant à lui sur l'étude du contenu ainsi que des différentes formulations du principe de contradiction dans le livre $\Gamma$ de la Métaphysique.

Après la science des causes et la science des êtres, c'est la science des substances, en tant que concept de la métaphysique aristotélicienne, qui constitue l'objet des contributions qui suivent. Dans «Substance et essence en Métaphysique Z, 6 : quelques observations $\gg$ (p. 113-131), David Charles examine les remarques d'Aristote aux chapitres 4 à 6 de Métaphysique $\mathrm{Z}$ sur la relation entre certaines substances et leur essence. Il analyse d'abord les apories qui résultent de l'idée répandue selon laquelle il s'agirait d'une identité numérique, pour arriver enfin à la conclusion que pour Aristote, la relation entre substance et essence dépend d'une forme particulière de la relation de prédication : celle dans laquelle l'essence fait qu'une chose est ce qu'elle est. Il n'y a donc pas lieu de dire ce qu'est une espèce indépendamment de son essence.

Les autres articles sur la science des substances portent sur le livre $\Lambda$ de la Métaphysique : celui de David Lefebvre, « La question de l'unité d'une science des substances: interprétations de Métaphysique, $\Lambda, 1,1069 \mathrm{a} 36-\mathrm{b} 2 »(\mathrm{p} .133-174)$, aborde la question de la relation entre physique et métaphysique en se concentrant (comme indiqué dans le titre) sur une phrase qui se trouve à la fin du chapitre $1 \mathrm{du}$ livre $\Lambda$. À la suite d'une analyse détaillée des questions textuelles (la leçon notamment des manuscrits $\mathrm{C}$ et $\mathrm{M}$ ), grammaticales et philosophiques liées à cette phrase, la traduction du passage proposée est la suivante : «Celles-là <les substances sensibles, corruptibles ou éternelles $>$ relèvent donc de la physique (car elles s'accompagnent du mouvement), mais celle-ci <la substance immobile> relève d'une autre <étude>, s'il est vrai que, pour eux, il n'existe aucun principe qui soit commun <scil. à ces deux sciences $>\gg$. Le propos d'Aristote ici ne serait donc pas 
d'affirmer la différence entre deux sciences, la physique et la philosophie première, mais de tirer avant tout les conséquences épistémologiques de la séparation de la substance immobile revendiquée par les platoniciens (1069a 33-34), ou encore de leur échec à rendre compte de la relation entre les substances immobiles et les êtres sensibles.

L'article de Jean-Baptiste Gourinat de son côté, « Le premier moteur selon Physique, VIII et Métaphysique, $\Lambda$ : physique et philosophie première » (p. 175206 avec un tableau comparatif en annexe), porte sur le traitement du premier moteur au livre VIII de la Physique et en Métaphysique $\Lambda$ et explique son apparition dans ces deux contextes différents. Dans les deux cas, dans $\Lambda 6$ comme dans le livre VII et la première partie du livre VIII de la Physique, Aristote adopte finalement l'hypothèse d'un premier moteur acte pur et immatériel, qui meut en tant que cause finale. En ce qui concerne les différences entre la façon dont le philosophe décrit le premier moteur dans la Physique et dans $\Lambda$, elles tiennent seulement à la différence entre un traitement physique qui étudie le premier moteur en tant que moteur, et le traitement métaphysique qui l'étudie en tant que substance.

La contribution de Suzanne Husson, «L'aporie d'une physique universelle: un commentaire de Parties des animaux I, 1, 641a32-b10 » (p. 207-237), aborde à son tour la question de la délimitation de l'objet propre d'une science, du côté cette fois de la physique. Dans le passage des Parties des animaux qui se trouve au centre de cette étude, Aristote, en appliquant à l'étude de l'âme le «principe de corrélation » (selon lequel l'étude des relatifs réciproques appartient à une même science), se trouve face à une conséquence inacceptable: une sorte de physique universelle qui engloberait tous les objets de la connaissance, dont ceux des mathématiques et de la science première. Afin d'y échapper, Aristote entreprend de montrer que l'intellect n'est pas une nature. Une solution plus simple et entièrement aristotélicienne (dont l'absence ici témoigne, selon l'auteur, du fait que le passage des Parties des animaux en question appartient à un premier état de la réflexion aristotélicienne) aurait été d'accepter que plusieurs sciences s'occupent du même objet, mais selon des caractéristiques différentes.

Cristina Viano, dans son article «États du corps, états de l'âme, "matière" du caractère : Aristote et la doctrine des qualités affectives » (p. 239-257), propose une analyse qui concerne de nouveau l'âme, cette fois en tant qu'objet de la physique. À travers l'analyse de la notion des qualités affectives au chapitre 8 des Catégories et en Physique VII, 3, est démontrée l'importance de l'élément matériel non seulement dans la constitution physique du corps mais aussi dans la formation du caractère moral. Plus précisément, sont posées et étudiées les questions suivantes : 1) comment les qualités affectives de l'âme fonctionnent-elles et à quel niveau agissent-elles ? 2) Comment naissent-elles ? 3) Quel rôle jouent-elles dans la détermination du caractère individuel de l'homme?

La dernière contribution de l'ouvrage est celle de Maddalena Bonelli, intitulée «Alexandre d'Aphrodise et la philosophie première » (p. 259-275). Sont analysés les deux passages principaux qui nous permettent de déterminer ce qu'Alexandre entend par «philosophie première » au sein de son commentaire de la Métaphysique d'Aristote. Ces deux passages semblent être en conflit : le premier (Alex., in Metaph., p. 245, 33-246, 13) présente deux philosophies premières, avec deux 
sens de «premier », tandis que le deuxième (Alex., in Metaph., p. 266, 2-14) établit de façon orthodoxe qu'il y en a seulement une et un seul sens de «premier $\gg$.

Pour conclure, cet ouvrage fait connaître à un public élargi les actes du séminaire du Centre Léon Robin, tout en constituant un instrument précis pour le travail spécialisé sur une série de questions cruciales pour la pensée aristotélicienne. La discussion en profitera sans doute beaucoup.

Pinelopi SKarsouli

CNRS, UPR 76, Centre Jean Pépin

Francesco VERDE, Elachista. La dottrina dei minimi nell'epicureismo, Louvain, Leuven University Press (De Wulf-Mansion Centre Series I), 2013, XIV + 440 pages, ISBN 9789058679475.

À un regard superficiel et peu averti, cet ouvrage pourrait sembler ressortir au genre du « presque tout sur presque rien » : un élément extrêmement pointu et technique de la doctrine épicurienne, la théorie des minima, devient l'objet d'une enquête qui, en 354 pages, va en épuiser toutes les dimensions : textuelle, doxographique, conceptuelle, systématique, historique, exégétique, etc. Mais il est entendu, en réalité, que le problème des elachista ne constitue en rien un détail de l'histoire de l'épicurisme, c'est même un apport essentiel de l'ouvrage de Francesco Verde (ci-après FV) que d'établir son importance de manière définitive.

L'idée de minimum semble fondatrice de l'atomisme en un premier sens: l'insécable représente en effet une limite à la réitération de l'opération de division d'un corps sensible. On peut considérer qu'une telle limite définit un minimum corporel, le plus petit élément physique assignable : l'atome (Lucrèce, I, 748 : in rebus minimum consistere). Lorsque Eusèbe de Césarée, citant Denys d'Alexandrie, nomme l'atome d'Épicure elachiston, peut-être, comme le suggère FV, commet-il une confusion entre l'atome lui-même et ses parties (p. 22-23), mais même si c'est le cas, celle-ci peut s'expliquer par une situation en elle-même équivoque. C'est cette complexité qui constitue le sujet même de l'ouvrage de FV. Elle tient à ce que, repoussant d'un cran l'assignation du minimum, Épicure a cru bon de distinguer des parties constitutives de l'atome lui-même. La nature de cette décision, son motif, sa portée, son destin historique sont systématiquement questionnés en trois grandes étapes, qui donnent lieu (I) à une analyse philologique et exégétique des sources premières de la théorie des minima : la Lettre à Hérodote d'Épicure et le De rerum natura de Lucrèce; (II) à une reconstitution du contexte historique de cette doctrine, essentiellement académicien, péripatéticien et mégarique; (III) à une étude de son développement à l'intérieur du Jardin, en relation avec les recherches géométriques dont témoignent plusieurs sources.

I. La lecture de Hrdt. 56-59 et de Lucrèce I, 599-634 et II, 481-499 est avant tout soucieuse d'inscrire ces textes dans une vision, non pas statique, mais dynamique de la doctrine épicurienne, c'est-à-dire de rester sensible aux évolutions possibles d'Épicure lui-même et de ses successeurs. Cette perspective continuera à prévaloir dans la suite de l'ouvrage. 